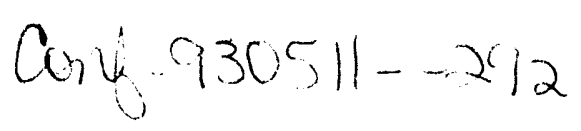

\title{
A mm-wave Planar Microcavity Structure for Electron Linear Accelerator System*
}

\author{
Y. W. Kang, H. Henlie $]^{\dagger}$ R. Kustom, F. Mills, and G. Mavrogenes \\ Accelerator Systems Division \\ Argonne National Laboratory \\ 9700 S. Cass Ave., Argonne, IL 60439
}

\section{Abstract}

The muffin-tin cavity structure is planar and well suited for $\mathrm{mm}$-wave accelerator with silicon etching techniques. A constant impedarice traveling-wave structure is considered for design simplicity. The RF parameters are calculated and the shunt impedance is compared with the shunt impedance of a disk loaded cylindrical structure.

\section{INTRODUCTION}

A double-sided muffin-tin structure was proposed for acceleration of relativistic electrons at $120 \mathrm{GHz}$ [1]. At very high frequencies, around $100 \mathrm{GHz}$, the standard technology for the fabrication of RF structures is no longer applicable. In recent years, micrometer silicon technology $[2,3]$ has become so well developed that largese-scale wafers in the range of $10 \mathrm{~cm}$ are available and, when metalized, could support planar RF structures for acceleration.

The structures can be made with high precision, on the order of one micrometer, and can be of nearly arbitrary shape. Glass fibers in V-grooves allow for alignment precisions of about 1 to 5 micrometers between wafers. Copper coating of these structures is well established and has been proven to withstand very high fields around $100 \mathrm{MV} / \mathrm{m}$ under DC conditions. Additionally, silicon has a very good thermal conductivity, only a factor 2.5 lower than copper at room temperature and even better than copper at liquid nitrogen temperature. Micro channels at the bottom of the wafer could therefore provide very effective cooling.

\section{Geometry and Mode of Operation}

The operating frequency we are considering is $120 \mathrm{GHz}$ (the wavelength $\lambda=2.5 \mathrm{~mm}$ ). For RF cavities of this size, planar structures are well suited. One such structure is the double-sided muffin-tin structure. The structure has a series of advantages:

- it is of simple geometry and best suited for microfabrication techniques,

- it is simple to cool from top and bottom,

- it has low higher-order modes which can be damped easily using the side openings,

- the side openings provide vacuum pumping slots in a natural way,

- Work supported by U.S. Department of Energy, Office of Basic Energy Sciences under Contract No. W-31-109-ENG-38.

TTechnische Universitaet, Institut fuer Theoretische Electrotechnik, Einstei nufer 17, 1000 Berlin 10, Germany

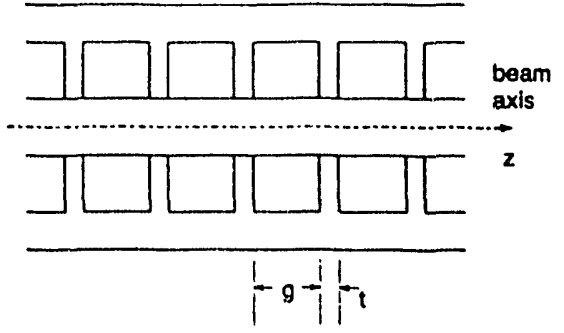

(a)

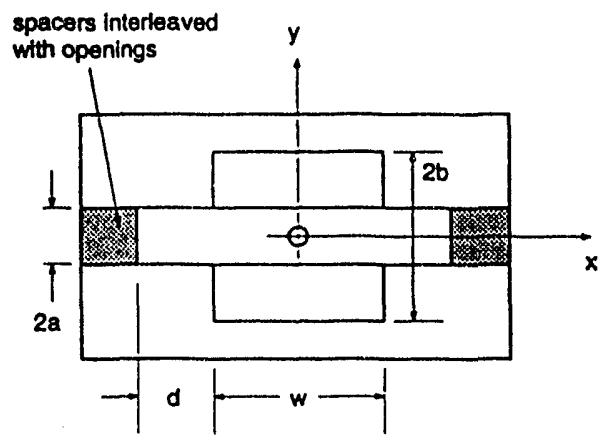

(b)

Figure 1

The double-sided muffin-tin structure. a) longitudinal b) (10) of this wo, for U. S. Government purposes. \section{transverse cut (a)}


Table 1

Comparison of 'TW shunt impedance and Q-factor of disk-loaded and muffin-tin designs.

\begin{tabular}{|l|c|c|c|c|}
\hline \multirow{2}{*}{$(\mathrm{mm})$} & \multicolumn{2}{|c|}{ Disk-loaded } & \multicolumn{2}{c|}{ Muffin-tin } \\
\cline { 2 - 5 } & $\begin{array}{c}r_{0} \\
(M \Omega / m)\end{array}$ & $\mathrm{Q}$ & $\begin{array}{c}r_{0} \\
(M \Omega / m)\end{array}$ & $\mathrm{Q}$ \\
\hline 0.04 & 441 & 2456 & 366 & 2460 \\
0.08 & 431 & 2401 & 358 & 2380 \\
0.12 & 417 & 2342 & 347 & 2310 \\
0.16 & 401 & 2280 & 335 & 2240 \\
0.20 & 385 & 2214 & 312 & 2160 \\
\hline
\end{tabular}

the filling time is shorter and the left-over RF power is taken out after the beam passage. This difference may be important, since due to the tininess of the structure the cooling will be at the limit of what is possible.

TW operation can be done in either a constant impedance (CI) structure or a constant gradient (CG) structure. For simplicity, we have chosen a CI structure. But, higher peak electrical fields and the non-uniform heat dissipation may either dictate the use of CG structures or split the structure in several sections of different but constant impedances. This is possible with different aspect ratios w/b (see Fig. 1) as will be shown in the next section. Other advantages a CG structure normally has as compared to a CI structure are lower sensitivity to errors in frequency, dimensions, and beam break-up. They are, we believe, less important since the muffin-tin is very open with correspondingly large coupling and small higher modes. In both cases, CI and CG, a $2 \pi / 3$ - mode will be used. This gives the highest shunt impedance, especially for thicker iris walls as are needed here.

Clearly the conclusions have to be reconsidered if the cooling problem turns out to be insurmountable or if the RF power available from the source is not high enough to reach the desired gradients of at least $10 \mathrm{MV} / \mathrm{m}$. If one has to go to cryogenic temperatures, SW operation may be preferable.

\section{RF PARAMETERS}

The cavity shunt impedances are calculated using the finite difference computer code MAFIA for the muffintin design in Figure 1 and for the $120 \mathrm{GHz} 2 \pi / 3$ - mode disk-loaded design [5], as shown in Table 1 . The shunt impedance of a disk-washer design at $120 \mathrm{GHz}$ is expected to be $\sim 390 \mathrm{M} \Omega / \mathrm{m}$ for $\mathrm{t}=0.12 \mathrm{~mm}$. (The $2.83 \mathrm{GHz}$ SLAC cavity has a shunt impedance of $\sim 60 \mathrm{M} \Omega / \mathrm{m}[6]$.)

For $120 \mathrm{GHz}(\lambda=2.5 \mathrm{~mm})$ the dimensions in $\mathrm{mm}$ were found to be

$$
\begin{aligned}
& a=0.3, \quad b=0.9 \\
& w=1.8, \quad d=0.8 \\
& g=0.633, \quad t=0.2 .
\end{aligned}
$$

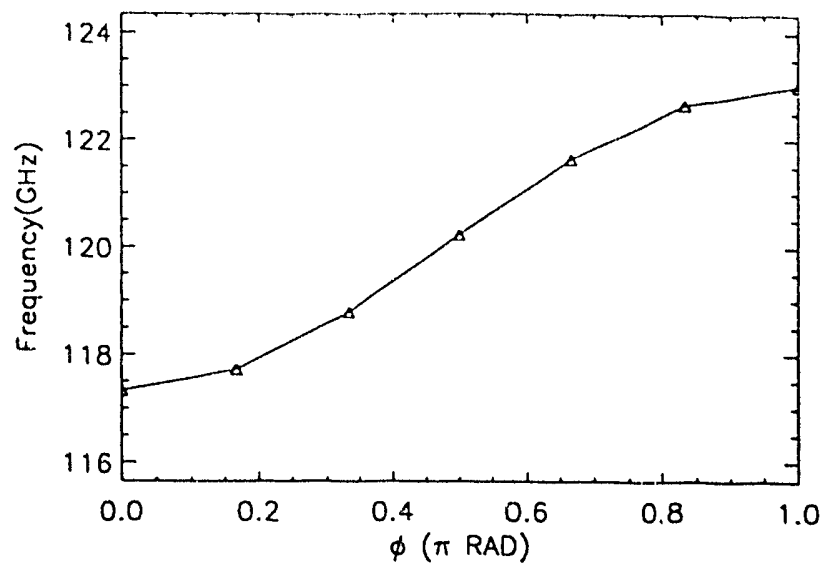

Figure 2

Dispersion relation of the muffin-tin structure with dimensions (2).

The period length $\mathrm{g}+\mathrm{t}$ was chosen for the $2 \pi / 3-$ mode

$$
2 \pi \frac{g+t}{\lambda}=\frac{2 \pi}{3}
$$

and the iris thickness $t$ fixed through practical reasons. The length of the side openings was taken large enough such that the fields have decayed sufficiently. The aperture $2 \mathrm{a}$ is in principle a free parameter and influences the passband bandwidth as well as Q-value, shunt impedance, and waktields. The value given above is a trade-off between the different requirements.

The dispersion relation is given in Fig. 2. It is well approximated by the theoretical cosine-shape

$$
\omega_{n} \simeq \omega_{\tau}\left(1-\frac{1}{2} k \cos \phi_{n}\right), \phi_{n}=\pi \frac{n}{N}, n=0,1,2, \ldots, N
$$

with $k=\frac{\omega_{N}-\omega_{0}}{\omega_{r}}=0.0475$ for $f_{r}=120 G H z$. From (4) we derive the group velocity as

$$
\frac{v_{g}}{c_{o}}=\frac{\partial \omega}{\partial \beta}=\frac{(g+t)}{c_{o}} \frac{\partial \omega}{\partial \phi}=\frac{\pi}{3} k \sin \frac{2 \pi}{3}=0.043 .
$$

Knowing the group velocity, the structure attenuation follows from

$$
\alpha=\frac{\omega_{r}}{2 v_{g} Q_{0}}=13.5 m^{-1} .
$$

A particle sitting on the crest of the RF wave gains an energy $V$ in a structure of length $\ell$

$$
V=\sqrt{2 \tau} \frac{1-e^{-\tau}}{\tau} \sqrt{r_{0} \ell P_{0}}, \quad \tau=\alpha \ell
$$

where $P_{o}$ is the input power. Since $r_{0}$ changes only slowly with the cavity dimensions, one can assume it to be constant and optimize $V$, yielding

$$
V_{\text {opt }} \simeq 0.9 \sqrt{r_{o} \ell P_{o}} \text { for } \tau_{\text {opt }}=1.26 \text {. }
$$




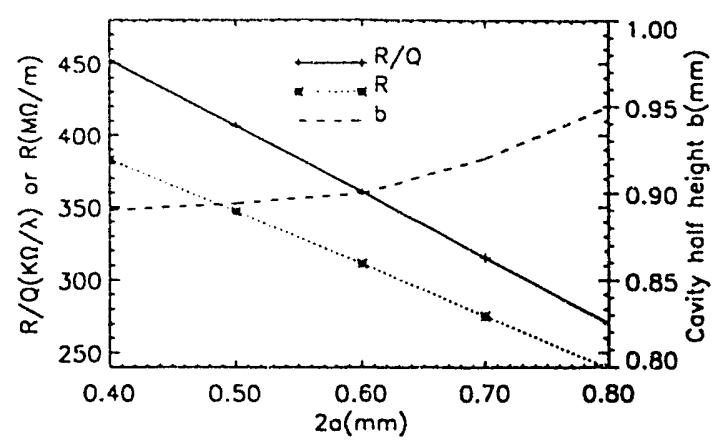

Figure 3

Shunt impedance, $r_{0} / Q$, and cavity depth $\mathrm{b}$ versus aperture $2 \mathrm{a}$ at constant cavity width $\mathrm{w}=1.8 \mathrm{~mm}$.

The optimum is very flat and not critically dependent on $\tau$. Substituting $\alpha$ from (6) into (8) gives $\ell=\tau_{\text {opt }} / \alpha=9.3$ $\mathrm{cm}$. This is about the maximum length one can reasonably achieve with silicon wafers. We choose it a little shorter

$$
\ell=7 \mathrm{~cm} \text { yielding } T=0.945
$$

to have resirve in the wafer length and also to have a somewhat bigher energy conversion. With this structure length we get

$$
V=4.1 \sqrt{P_{o}} K V .
$$

The ratios of input to output gradient and power loss are

$$
\frac{E_{(z=0)}}{E_{(z=\ell)}}=2.6, \quad \frac{P_{(z=0)}^{\prime}}{P_{(z=\ell)}^{\prime}}=6.6
$$

and the number of cells per section is $N=\ell /(g+t)=84$. To get an accelerating gradient $E=V / \ell$ of $10 \mathrm{MV} / \mathrm{m}$ we need, following Eq. (10), a peak input power of

$$
P_{0}=\left(\frac{E \ell}{4.1 \times 10^{3}}\right)^{2}=29.1 \mathrm{KW} \text {. }
$$

For small beam currents one may tolerate even smaller apertures than the chosen $2 a=0.6 \mathrm{~mm}$. In that case the shunt impedance goes up as shown in Fig. 3.

If it turns out that the strong dependence of power dissipation along the structure, Eq. (11), is not tolerable for cooling reasons, we can choose a quasi CG structure with a few CI subsections. The energy gain, Eq. (10), will only change marginally but the power dissipation can be made nearly uniform along the structure. Fig. 4 shows the necessary aspect ratio $\mathrm{w} / \mathrm{b}$ in order to adjust the coupling constant $k$ and, therefore, $v_{g}$ (see Eq. 5).

The current threshold for the regenerative multi-bunch beam break-up was estimated as [3]

$$
I_{t h} \simeq \pi \frac{\left|v_{g}\right|}{c_{o}} \frac{1}{\lambda r_{\perp} / Q} \frac{p c_{o}}{e}\left(\frac{\lambda}{2 \ell}\right)^{3} .
$$

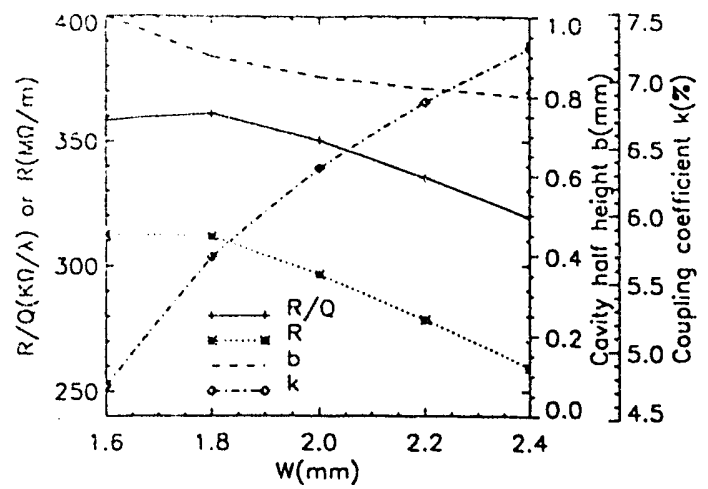

Figure 4

Shunt impedance $r_{o}, r_{o} / Q$, coupling constant $\mathrm{k}$, and cavity depth $\mathrm{b}$ versus cavity width $\mathrm{w}$ at constant aperture $2 \mathrm{a}=0.6 \mathrm{~mm}$.

If the deflecting $H E M_{11}$ mode is around $150 \mathrm{GHz}$ $(\lambda=2 \mathrm{~mm})$, beam energy $p c_{o}=10 \mathrm{MeV}, v_{g}=0.05 c_{o}$, and $\lambda r_{\perp} / Q=200 \Omega$, the current threshold is $23 \mathrm{~mA}$.

\section{Conclusion}

A double-sided muffin-tin structure is ideally suited for mm-wave frequencies and microfabrication techniques. A traveling wave mode with a $2 \pi / 3$ phase advance per cell has adequate RF parameters and could even form an approximated constant gradient structure. In addition, it has transverse fields of quadrupole character which can be used, at least partially, for focusing.

\section{REFERENCES}

[1] H. Henke, Y. W. Kang, and R. L. Kustom, "A mmwave RF structure for relativistic electron acceleration," APS Note MMW-1, September 1992.

[2] H. Guckel, K. J. Skrobis, T. R. Christenson, J. Klein, S. Han, B. Choi, and E. G. Lovell, "Fabrication of Assembled Micromechanical Components via Deep XRay Lithography," MEMS 1991, Nara, Japan, Jan. 1991.

[3] R. T. Howe, R. S. Muller, K. Y. Gabriel, and W. S. N. Trimmer, IEEE Spectrum, p. 29, July 1990.

[4] G. A. Loew and R. B. Neal, Linear Accelerators, chapters B.1.1 and B.1.4, North-Holland Publishing Co., 1970.

[5] R. Klatt, F. Krawczyk, W. R. Novender, C. Palm, B. Steffen, T. Weiland, T. Barts, M. F. Browman, R. Cooper, C. T. Mottershead, G. Rodenz, and S. G. Wipf, Proc. of the 1986 Linear Acc. Conf., SLACReport-303, Sept. 1986.

[6] G. A. Loew, R. H. Miller, R. A. Early, and K. L. Bane, "Computer Calculations of Traveling-Wave Periodic Structure Properties," IEEE Trans. on Nuclear Science, Vol. NS-26, No. 3, June, 1979. 

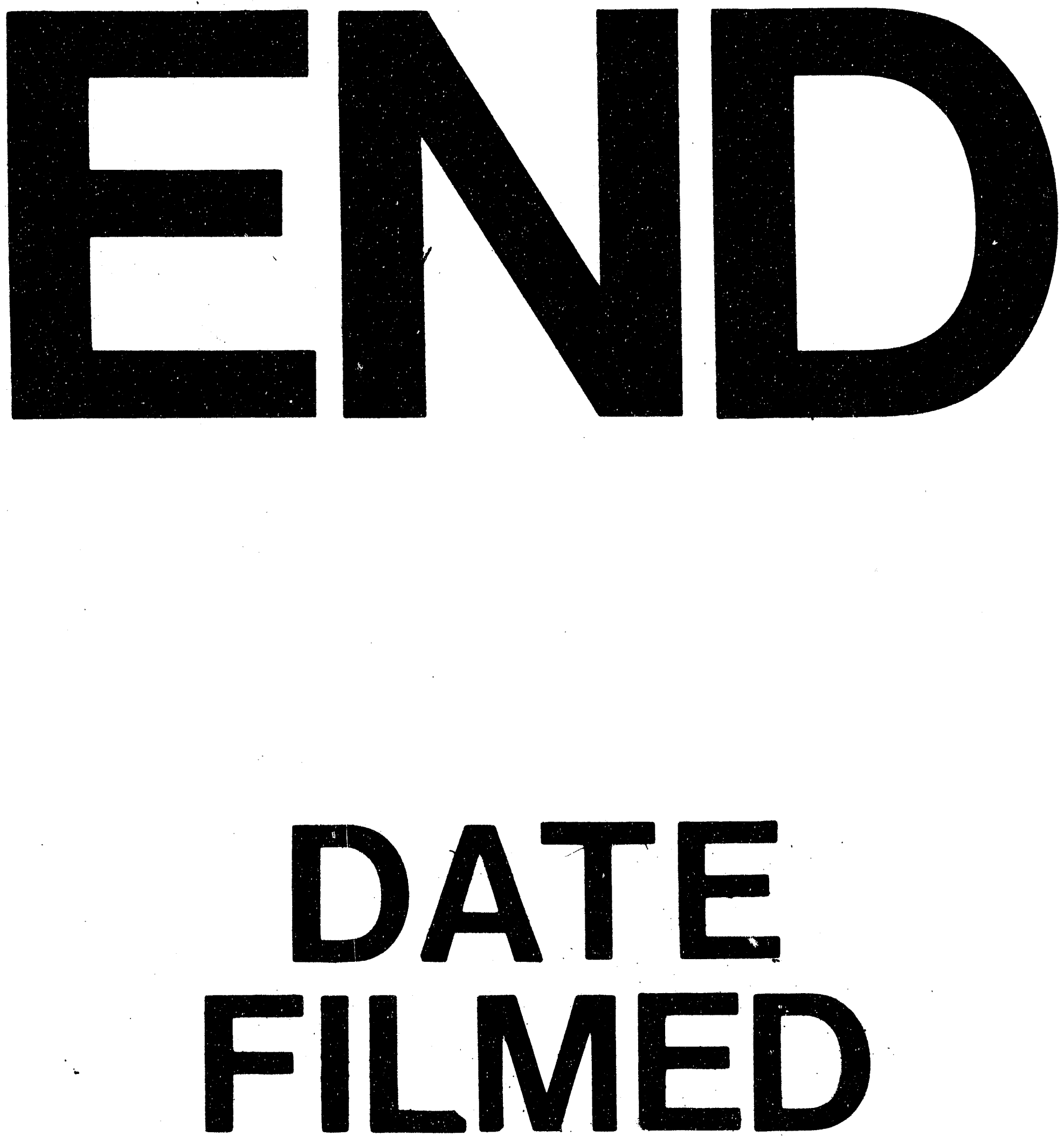

I

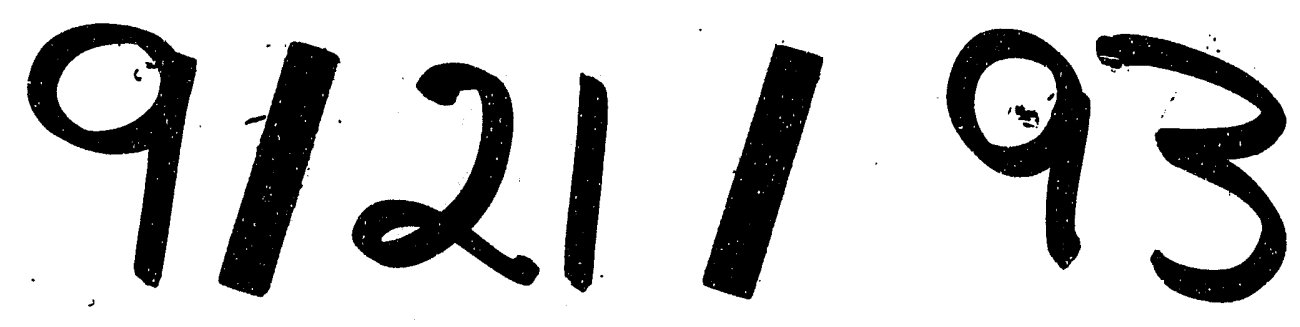


